\title{
Complete response to low-dose sorafenib in a patient with metastatic renal cell carcinoma: A case report
}

\author{
Jun Morita, MD;" Michio Naoe, MD; ${ }^{*} Y u$ Ogawa, MD; Takehiko Nakasato, MD;" Motoko Sugahara, MD;* \\ Masashi Morita, MD; Kohzo Fuji, MD; ${ }^{*}$ Takashi Fukagai, MD; ${ }^{*}$ Haruaki Sasaki, MD; ${ }^{\dagger}$ Yoshio Ogawa, MD*
}

*Department of Urology, Showa University, School of Medicine, Tokyo, Japan; †Department of Urology, Showa University, Fuijgaoka Hospital, Yokohama, Japan

Cite as: Can Urol Assoc J 2013;7(5-6):e351-4. http://dx.doi.org/10.5489/cuaj.1210 Published online May 13, 2013.

\section{Abstract}

We present a case of a patient with metastatic renal cell carcinoma (mRCC) who was treated solely with low-dose sorafenib and achieved a complete response (CR). A 79-year-old man with cytokine-refractory $\mathrm{mRCC}$ involving the lung, abdominal wall and lymph nodes was treated with low-dose sorafenib (400 mg/day) as a second-line therapy. Five months into treatment, CR was confirmed by follow-up computed tomography. No severe adverse events were observed and sorafenib treatment was continued without appearance of new lesions. Although sorafenib has been approved for mRCC treatment, complete clinical recovery is uncommon and has rarely been described. In this case, low-dose sorafenib appears to be sufficient for achieving CR while suppressing toxicity. Furthermore, long-term continuous administration induces the patient to obtain disease stabilization. However, considering toxicity and treatment costs, it is debatable whether treatment should be discontinued or sustained after CR.

\section{Introduction}

The development of molecular-targeted therapies has changed the treatment approach for metastatic renal cell carcinoma (mRCC). New anti-angiogenic agents, such as tyrosine kinase inhibitors (TKIs), promote disease stabilization and increase progression-free survival (PFS) rates, but complete response (CR) is rare. One of these agents, sorafenib, exhibits anti-tumour activity as a second-line therapy in patients with cytokine-refractory, clear-cell mRCC. In this report, we describe a patient with $\mathrm{mRCC}$ who achieved CR with low-dose sorafenib therapy alone.

\section{Case report}

A 79-year-old man presented with an episode of transient unconsciousness and was diagnosed with transient ischemic attack (TIA) by his primary care physician. At that time, a whole-body computed tomography (CT) scan incidentally revealed a right renal mass measuring over $5 \mathrm{~cm}$. The mass appeared to be confined within Gerota's fascia, but had renal vein tumour embolism. He visited our hospital to undergo open right radical nephrectomy. Histopathological examination revealed RCC (clear cell carcinoma, G2, INF $\alpha$, pT3bNOM0).

Six months after surgery, a follow-up CT revealed a pulmonary metastatic lesion. We had hoped to perform an additional surgical treatment for this patient. However, considering the metastasis was only in the lung, the patient chose the treatment with interferon (IFN)- $\alpha$. More than 10 months later, additional multiple metastatic lesions appeared in the lung, abdominal wall and para-aortic lymph nodes (Fig. 1a, Fig. 1b). A needle biopsy of the abdominal wall lesion revealed clear cell carcinoma.

We initiated treatment with sorafenib (400 mg/day) for mRCC, because the patient did not have adequate cardiac function for the sunitinib. Dosage of sorafenib was determined based on age (79 years old), performance status (grade 2 according to the Eastern Cooperative Oncology Group [ECOG]), and weight (51.6 kg). According to the Memorial Sloan-Kettering Cancer Center (MSKCC) prognostic criteria, this patient was poor-risk (Karnofsky performance status $60[<80]$; time from diagnosis to treatment 6 months [ $<12$ months]; Hb $11.1 \mathrm{~g} / \mathrm{dL}[<14.0-18.0]$, lactic dehydrogenase $185 \mathrm{IU} / \mathrm{L}$ [105-220], Ca [corrected] $9.9 \mathrm{mg} / \mathrm{dL}$ [>10.0]). Furthermore, according to the Heng criteria, the patient was also poor-risk. We observed hypertension (grade 1 according to the Common Terminology Criteria for Adverse Events [CTCAE]) 1 week into the sorafenib administration, which was not severe and improved without any treatment, immediately. After 7 weeks of sorafenib, hand-foot skin reactions (grade 2) were observed and treated with topical steroid ointment. Other adverse events were stomatitis (grade 1) and fatigue (grade 1), but no severe toxicity was observed. One month into the sorafenib administration, a CT showed disappearance of lung metastasis and shrinkage of other 


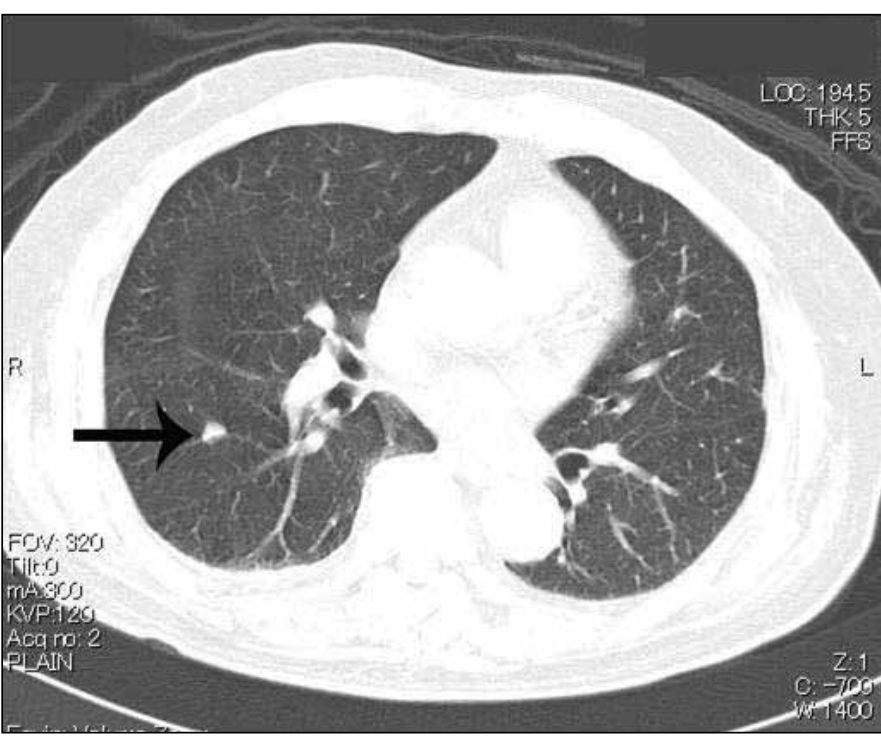

Fig. 1a. A follow-up computed tomography scan revealed a pulmonary metastatic lesion.

metastatic lesions. Furthermore, a CT at 3 months showed disappearance of all metastatic lesions and a CT at 2 months later showed no relapse of metastasis (Fig. 2a, Fig. 2b). Thus, in consultation with radiologists, we evaluated this case as CR according to the Response Evaluation Criteria in Solid Tumors (RECIST).

There were no severe adverse events that caused us to consider reducing sorafenib dosage or discontinuing its administration. Hand-foot skin reactions were mild in degree and the patient was able to manage treatment by himself. Nevertheless, taking the medication and managing the handfoot skin reactions gradually made the patient feel burdened and annoyed. After confirming CR, the patient proposed reducing the dosage by half ( $200 \mathrm{mg} /$ day). Follow-up CTs were performed every 2 months. Nine months have passed since initiation of sorafenib treatment, without relapse of metastasis. We plan to continue the reduced dose as maintenance therapy.

\section{Discussion}

The prognosis of patients with mRCC is generally poor, with a 1 to 2 year survival rate of only $10 \%$ to $20 \%$. About a third of patients present with metastatic disease at the time of initial diagnosis, and another $25 \%$ will develop metastasis at a later stage. ${ }^{1}$ Until recently, standard treatment for mRCC was immunotherapy with IFN- $\alpha 2 \mathrm{a}$ and/or interleukin (IL)-2. However, the efficacy of these agents has not been sufficient and they are associated with significant toxicity.

Treatment strategy for mRCC changed with the development of multitargeted tyrosine kinase inhibitors (TKIs). Sorafenib (Nexavar, Bayer Schering Pharma, Berlin, Germany) is an oral, multitargeted TKI that targets prolifera-

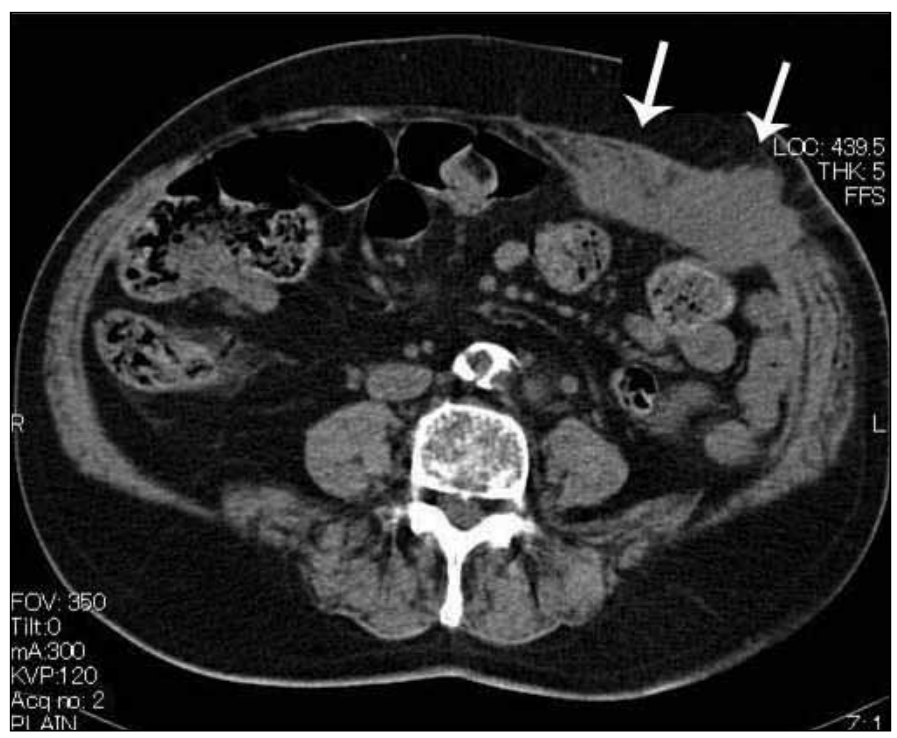

Fig. $\mathbf{1 b}$. An additional metastatic lesion appeared in abdominal wall.

tion and angiogenesis by inhibiting tyrosine kinases including Raf-1, wild-type and mutant B-Raf, vascular endothelial growth factor receptor (VEGFR)-1, VEGFR-2, VEGFR-3, platelet derived growth factor receptor (PDGFR)- $\beta$, c-KIT protein, fms-like tyrosine kinase-3 (FLT-3), and RET receptor. ${ }^{2,3}$ In a phase III trial, sorafenib was effective in lengthening PFS time in patients with advanced RCC compared with the placebo group (5.5 months vs. 2.8 months). ${ }^{4}$ The major benefits of TKIs are considered to be promoting disease stabilization and increasing PFS.

Thus, although TKIs have been approved for mRCC treatment, unfortunately, complete clinical recovery is uncommon in general cases and has been rarely described. Reported CR rates are $<1 \%$, for instance, 0/97 patients in a phase II trial, ${ }^{5} 1 / 451$ in a phase III trial in the United States, ${ }^{4}$ and 0/129 in a Japanese phase II study. ${ }^{6}$ Nevertheless, CR is the most favourable outcome from the patient perspective. TKIs have the potential of achieving CR in some rare cases, thus details of such a case achieved with sorafenib are important to discuss.

A few cases of CR achieved with sorafenib therapy have been reported. To our knowledge, only 4 cases have been described in detail, with additional cases reported with limited description..$^{7-9} \mathrm{~A}$ recent report discussed the possibility of achieving CR by combining neoadjuvant sorafenib with surgical resection. ${ }^{10}$ TKIs may induce partial carcinoma shrinkage and substitution with necrotic-fibrotic tissue, allowing easier resection of smaller, limited lesions. Thus, this combination therapy may certainly lead to durable remission in selected cases. Indeed, Staehler and colleauges have reported a patient with CR after sorafenib administration and surgical resection; this patient was free of disease for over 29 months. $^{7}$ As for TKIs (sorafenib and sunitinib) 


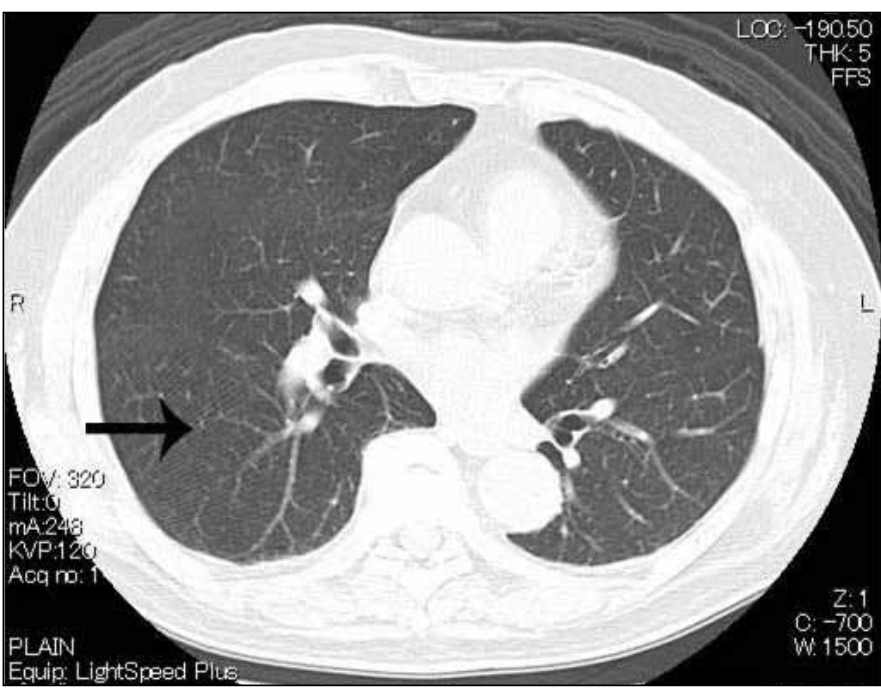

Fig. 2a. A follow-up computed tomography of this patient was performed without enhanced, because of allergic history.

and combining surgery, there are large series of patients with mRCC achieving CR during TKIs treatment, whereas agents of TKIs are mainly sunitinib. ${ }^{11,12}$ One series included 36 patients who achieved CR during TKI treatment; in this series, 12 patients (33\%) remained recurrence free at the 12-month median follow-up. ${ }^{11}$ In another series, 64 patients achieved CR during TKI treatment. Of the total 64 patients, 29 remained recurrence free at 8.5 months (or 255 days). ${ }^{12}$

Our case is a unique one of CR in a patient with $\mathrm{mRCC}$ treated solely with low-dose sorafenib. Furthermore, the patient was poor-risk according to the MSKCC prognostic criteria. In their series of 64 patients, Albiges and colleagues reported that despite the fact that most patients were in the favourable or intermediate risk group, 3 patients at poor risk also achieved CR. ${ }^{12}$ Sorafenib, $800 \mathrm{mg}$, is generally a good dosage; this is the case at our institution. Nevertheless, clear criteria for the initial dosage, considering condition of the patient, has been rarely described. In this case, to suppress the expression of adverse events and after consulting the patient, we determined $400 \mathrm{mg}$ for this patient based on age, performance status and weight. For TKIs, longterm continuous administration, while minimizing adverse events, has been suggested to be important in achieving CR. ${ }^{12}$ Continuous sorafenib administration may induce several adverse events; the most common are dermatologic symptoms and diarrhea. ${ }^{4}$ Although hand-foot skin reactions were observed in this case, it was not severe and could be managed by the patient. The low dose may account for the mild degree of adverse events in this patient. In addition, adverse events were noticed at an early stage and treated promptly, allowing continued sorafenib treatment. We believe this patient achieved CR because of the continuous administration of sorafenib, rather than the low dose.

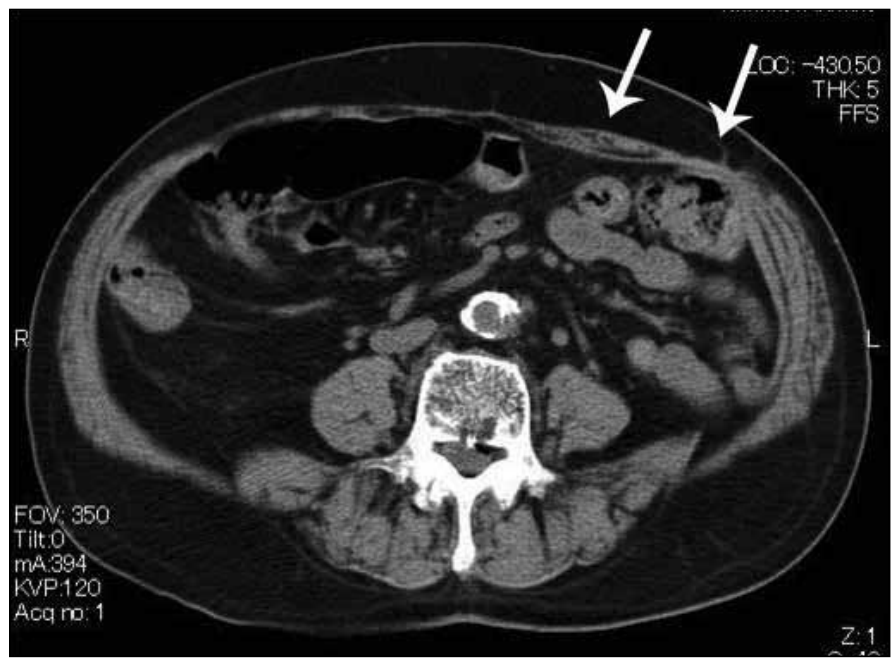

Fig. $\mathbf{2 b}$. A computed tomography scan at 3 months showed disappearance of all metastatic lesions.

Given that the clinical benefits of TKIs are achieving a partial response and long-term stabilization, CR may not be an important outcome to consider. Even in a CR, the risk of developing new lesions and recurrence still exist. While TKIs may be effective in the weeks after treatment initiation, rapid regrowth of tumours and metastasis may occur in some cases following their discontinuation, known as the rebound phenomenon. ${ }^{8}$ Little is known about how long therapy must be continued. Moreover, it is controversial as to the dosage reductions after achieving CR; these issues must be considered. RECIST criteria may be insufficient to understand the actual disease state since microscopic metastatic lesions may remain. Indeed, Johannsen and colleagues have reported a case of CR with recurrence after treatment discontinuation. ${ }^{8}$ However, discontinuation and dose reduction have advantages, including avoidance of adverse events, improvement in quality of life and reduction of treatment cost.

Although achieving CR is favourable for the patient, medication adherence may potentially suffer when a patient is informed of CR. On the other hand, other patients willingly continue taking the medication that led to CR. In these situations, physicians need to explain the necessity of treatment and risks of discontinuation in addition to the advantage of discontinuation. If a patient selects treatment continuation, we should continue treatment with an optimal dose, and monitor the patient's condition and adverse events.

\section{Conclusion}

This case illustrates that it is possible to achieve CR with low-dose TKIs. However, it is debatable whether patients should continue TKI treatment after achieving CR. It is possible that, when CR is achieved, continuous administration of TKIs might contribute to long-term disease stabilization, 
Morita et al.

even with a dosage low enough to suppress toxicity. On the contrary, treatment discontinuation with TKIs after CR might be effective. More research is needed.

Competing interests: None declared.

This paper has been peer-reviewed.

\section{References}

1. Jemal A, Siegel R, Ward EA, et al. Cancer Statistics, 2008. CA Cancer J Clin 2008;58:71-96. http:// dx.doi.org/10.3322/CA.2007.0010

2. Wilhelm SM, Carter C, Tang L, et al. BAY 43-9006 exhibits broad spectrum oral antitumor activity and targets the Raf/MEK/ERK pathway and receptor tyrosine kinases involved in tumor progression and angiogenesis. Cancer Res 2004;64:7099-109. http://dx.doi.org/10.1158/0008-5472.CAN-04-1443

3. Carlomagno F, Anaganti S, Guida T, et al. BAY 43-9006 inhibition of oncologic RET mutants. J Natl Cancer Inst 2006;98:326-34. http://dx.doi.org/10.1093/inci/dij069

4. Escudier B, Eisen T, Stadler WM, et al. Sorafenib in advanced clear-cell renal-cell carcinoma. N Engl J Med 2007;356:125-34. http://dx.doi.org/10.1056/NEJMoa060655

5. Escudier B, Szczylik C, Hutson T, et al. Randomized phase II trial of first-line treatment with sorafenib versus interferon alfa-2a in patients with metastatic renal cell carcinoma. J Clin Oncol 2009;27-8:1280-9. http://dx.doi.org/10.1200/JC0.2008.19.3342
6. Akaza H, Tsukamoto $\mathrm{T}$, Murai $\mathrm{M}$, et al. Phase II study to investigate the efficacy, safety, and pharmacokinetics of sorafenib in Japanese patients with advanced renal cell carcinoma. Jpn I Clin Oncol 2007;37:755-62. http://dx.doi.org/10.1093/iico/hym095

7. Staehler $M$, Haseke $N$, Zilinberg $E$, et al. Complete remission achieved with angiogenic therapy in metastatic renal cell carcinoma including surgical intervention. Uro Oncol 2010;28:139-44. http://dx.doi. org/10.1016/i.urolonc.2009.03.033

8. Johannsen M, Flörcken A, Bex A, et al. Can tyrosine kinase inhibitors be discontinued in patients with metastatic renal cell carcinoma and a complete response to treatment? A multicentre, retrospective analysis. Eur Urol 2009;55:1430-9. http://dx.doi.org/10.1016/i.eururo.2008.10.021

9. Benincasa $E$, Conti A, Bossone $G$, et al. A case of complete clinical response with sorafenib in a patient with thyroid gland metastases from renal cell carcinoma 17 years from diagnosis. Tumori 2009;95:403.

10. Kinoshita T, Inove H, Kinouchi T, et al. Preoperative induction with sorafenib pathologically downstaged advanced renal cell carcinoma: A case report. Int J Urol 2010;17:286-8. http://dx.doi.org/10.1111/ j.1442-2042.2009.02444.x

11. Johannsen $M$, Staehler $M$, Ohlmann $C H$, et al. Outcome of treatment discontinuation in patients with metastatic renal cell carcinoma and no evidence of disease following targeted therapy with or without metastasectomy. Ann Oncol 2011;22:657-63. http://dx.doi.org/10.1093/annonc/mdq437

12. Albiges $L$, Oudard $S$, Negrier $S$, et al. Complete remission with tyrosine kinase inhibitors in renal cell carcinoma. J Clin Oncol 2012;30-5:482-7.

Correspondence: Dr. Jun Morita, Department of Urology, Showa University, School of Medicine, 1-5-8, Hatanodai, Shinagawa-ku, Tokyo, 142-8666, Japan; fax: +81-3-3784-1400; moritajun@hotmail.co.jp 